Bull. Austral. Math. Soc.

$46 \mathrm{~F} 05,60 \mathrm{~B} 11$

VOL. 42 (1990) [125-131]

\title{
CAUCHY REPRESENTATION OF DISTRIBUTIONS AND APPLICATIONS TO PROBABILITY
}

\section{Aslam Chaudhry}

The Cauchy representation is not valid for every Schwartz distribution $f \in(D(R))^{\prime}$ because $(t-z)^{-1} \notin D(R)$. Since for all $z \in\{z: \operatorname{Im} z \neq 0\}$ the kernel $(t-z)^{-1}$ belongs to $D_{L_{p}}(R)(1<p<\infty)$, the Cauchy representation of the distributions in $\left(D_{L},(R)\right)^{\prime}$ seems possible.

In this paper, we prove this fact. It is also proved that every probability density defines a generalised function on the space $D_{L_{p}}(R)(1<p<\infty)$ of test functions. Applications of these results in probability theory are discussed.

\section{INTRODUCTION}

Let $f \in\left(D_{L_{p}}(R)\right)^{\prime}$. If there exists a complex valued function $F(z)$ analytic in the $z$-plane except possibly on the real axis $\{z: \operatorname{Im} z=0\}$ such that

$$
\lim _{\varepsilon \rightarrow 0^{+}} \int_{-\infty}^{\infty}[F(x+i \varepsilon)-F(x-i \varepsilon)] \phi(x) d x=\langle f, \phi\rangle
$$

for all $\phi \in D_{L_{p}}(R)$, then $F(z)$ is called the analytic representation of $f \in\left(D_{L_{p}}(R)\right)^{\prime}$. The Cauchy integral representation of analytic functions (in terms of their boundary values) is an important tool for the mathematical study of physical phenomena. The role of such representations is even more important when we consider the generalised functions.

The analytic representation of a generalised function $f$ with compact support is given by [1]

$$
\widehat{f}(z)=\frac{1}{2 \pi i}\left\langle f(t), \frac{1}{t-z}\right\rangle .
$$

$\widehat{f}(z)$ is called Cauchy representation of $f \in(E(R))^{\prime}$. The Cauchy representation (2) is not valid for every Schwartz distribution $f \in(D(R))^{\prime}$ because $(t-z)^{-1} \notin D(R)$. For all $z \in\{z: \operatorname{Im} z \neq 0\}$ the kernel $(t-z)^{-1}$ belongs to $D_{L_{p}}(R)(1<p<\infty)$, thus the Cauchy representation of the generalised functions in $\left(D_{L_{p}}(R)\right)^{\prime}$ seems possible. We prove this fact and construct an example of the Cauchy representation of generalised functions with non-compact support. Since $\left(D_{L_{p}}(R)\right)^{\prime} \supset(E(R))^{\prime}$ we extend the result to a wider class of generalised functions proved in [3].

Received 25 September 1989

The author is thankful to late Professor M.H. Kazi for his helpful comments and suggestions.

Copyright Clearance Centre, Inc. Serial-fee code: 0004-9729/90 \$A2.00+0.00. 


\section{The testing function SPaCe $D_{L_{p}}(R),(1<p<\infty)$}

An infinitely differentiable function $\phi$ defined over $R$ is said to belong to the space $D_{L_{p}}(R)$ if $\phi^{(k)}(x)$ belongs to $L_{p}(R)$ for each $k=0,1,2,3, \ldots$ We define a sequence of semi-norms $\left\{\gamma_{m}\right\}_{m=0}^{\infty}$ on $D_{L_{p}}(R)$ as follows: For $\phi \in D_{L_{p}}(R)$ we define

$$
\gamma_{m}(\phi)=\left[\int_{-\infty}^{\infty}\left|\phi^{m}(x)\right|^{p} d x\right]^{1 / p} \quad m=0,1,2,3, \ldots
$$

Since $\gamma_{0}$ is a norm, the sequence of semi-norms $\left\{\gamma_{m}\right\}_{m=0}^{\infty}$ is separating [6]. A sequence $\left\{\phi_{\mu}\right\}_{\mu=1}^{\infty}$ in $D_{L_{p}}(R)$ is said to converge to $\phi \in D_{L_{p}}(R)$ if for each $m=0,1,2,3, \ldots$,

$$
\gamma_{m}\left(\phi_{\mu}-\phi\right) \longrightarrow 0 \text { as } \mu \longrightarrow \infty
$$

The space $D_{L_{p}}(R)$ is multinormed and complete $[2,5]$. Moreover, the Schwartz space $D(R)$ of test functions is dense in $D_{L_{p}}(R)[2]$. We denote by $\left(D_{L_{p}}(R)\right)^{\prime}$ the space of all continuous linear functionals on $D_{L_{p}}(R)$. We state the structure formula for $f \in\left(D_{L_{p}}(R)\right)^{\prime}[\mathbf{4}, \mathbf{5}]$.

THEOREM 1. If $f \in\left(D_{L_{p}}(R)\right)^{\prime}(1<p<\infty)$, then $f$ is equal to a finite linear combination of the derivatives of functions in $L_{q}(R)$, that is, for each $f \in\left(D_{L_{p}}(R)\right)^{\prime}$

$$
\langle f, \phi\rangle=\sum_{\alpha=0}^{r}(-1)^{\alpha} \int_{-\infty}^{\infty} f_{|\alpha|}(x) \phi^{(\alpha)}(x) d x \quad \forall \phi \in D_{L_{p}}(R)
$$

where $f_{|\alpha|}$ are functions in $L_{q}(R), 1 / p+1 / q=1$.

THEOREM 2. Let $f \in\left(D_{L_{p}}(R)\right)^{\prime},(1<p<\infty)$ and $F(z)$ be the complex-valued function defined in the region $\Omega=\{z: \operatorname{Im} z \neq 0\}$ by:

$$
F(z)=\frac{1}{2 \pi i}\left\langle f(t), \frac{1}{t-z}\right\rangle
$$

Then $F(z)$ is the Cauchy representation of the generalised function $f$.

Proof: To prove the analyticity of $F(z)$ we note that

$$
\frac{1}{h}[F(z+h)-F(z)]=\frac{1}{2 \pi i}\left\langle f(t), \frac{1}{h}\left[\frac{1}{t-z-h}-\frac{1}{t-h}\right]\right\rangle,
$$

and we prove that

$$
h^{-1}\left[(t-z-h)^{-1}-(t-h)^{-1}\right] \text { converges to }(t-z)^{2}
$$


in the sense of convergence in $D_{L_{p}}(R)$.

Assume that

$$
\Delta_{h}(t, z)=\left[h^{-1}\left\{(t-z-h)^{-1}-(t-z)^{-1}\right\}-(t-z)^{-2}\right] .
$$

Then for $\operatorname{Im} z=y \neq 0$,

$$
\begin{gathered}
\left\|\frac{d^{k} \triangle_{h}(t, z)}{d t^{k}}\right\|_{L_{p}} \leqslant h O\left(|y|^{k+3}\right) \rightarrow 0 \text { as } h \rightarrow 0 \\
(k=0,1,2,3, \ldots)
\end{gathered}
$$

so that

$$
\gamma_{k}\left(\triangle_{h}(-, z)\right) \rightarrow 0 \quad(k=0,1,2,2, \ldots)
$$

as $h \rightarrow 0$. Therefore,

$$
\frac{d}{d z} F(z)=\left\langle f(t), \frac{1}{(t-z)^{2}}\right\rangle \quad \forall z \in \Omega
$$

Thus $F(z)$ is analytic in the region $\Omega$. Let us define

$$
I(\varepsilon)=\int_{-\infty}^{\infty}[F(x+i \varepsilon)-F(x-i \varepsilon)] \phi(x) d x .
$$

Since $\phi \in D_{L_{p}}(R)$ and for each fixed $\varepsilon>0, F(x+i \varepsilon) \in L_{q}(R)[1 / p+1 / q=1]$, the integral (7) exists for all $\varepsilon>0$. From (5) and (7) we get:

$$
\begin{aligned}
I(\varepsilon) & =\frac{1}{2 \pi i} \int_{-\infty}^{\infty} \phi(x)\left\langle f(t),\left[\frac{1}{t-x-i \varepsilon}-\frac{1}{t-x+i \varepsilon}\right]\right\rangle d x \\
& =\int_{-\infty}^{\infty} \phi(x)\left\langle f(t), \frac{\varepsilon}{\pi\left[(t-x)^{2}+\varepsilon^{2}\right]}\right\rangle d x .
\end{aligned}
$$

Using the structure formula (4) for $f$ we get

$$
I(\varepsilon)=\sum_{j=1}^{T}(-1)^{j} \int_{-\infty}^{\infty}\left\langle f_{j}(t), \frac{\partial^{j}}{\partial t^{j}}\left[\frac{\varepsilon}{\pi\left[(t-x)^{2}+\varepsilon^{2}\right]}\right]\right\rangle \phi(x) d x
$$

where $f_{j} \in L_{q}(R)(1 \leqslant j \leqslant r)$. However,

$$
\begin{aligned}
\frac{\partial^{j}}{\partial t^{j}}\left[\frac{\varepsilon}{\pi\left[(t-x)^{2}+\varepsilon^{2}\right]}\right]=(-1)^{j} \frac{\partial^{j}}{\partial x^{j}}\left[\frac{\varepsilon}{\pi\left[(t-x)^{2}+\varepsilon^{2}\right]}\right] \\
(\forall j=1,2,3, \ldots)
\end{aligned}
$$


Therefore,

$$
I(\varepsilon)=\sum_{j=1}^{r}(-1)^{j} \int_{-\infty}^{\infty}\left\langle f_{j}(t),(-1)^{j} \frac{\partial^{j}}{\partial x^{j}}\left[\frac{\varepsilon}{\pi\left[(t-x)^{2}+\varepsilon^{2}\right]}\right]\right\rangle \phi(x) d x
$$

Using Fubini's theorem and integration by parts we get

$$
I(\varepsilon)=\sum_{j=1}^{r}\left\langle f_{j}(t), \frac{\varepsilon}{\pi} \int_{-\infty}^{\infty} \frac{\phi^{(j)}(x) d x}{\left[(t-x)^{2}+\varepsilon^{2}\right]}\right)
$$

However,

$$
\frac{\varepsilon}{\pi} \int_{-\infty}^{\infty} \frac{\phi^{(j)}(x) d x}{\left[(t-x)^{2}+\varepsilon^{2}\right]} \rightarrow \phi^{(j)}(t) \text { in } D_{L_{p}}(R) \text { as } \varepsilon \rightarrow 0^{+}
$$

see $[2,5,6]$.

Hence, letting $\varepsilon \rightarrow 0^{+}$in (8) we get:

$$
\begin{aligned}
\lim _{\varepsilon \rightarrow 0^{+}} I(\varepsilon) & =\sum_{j=1}^{r}\left\langle f_{j}(t), \phi^{(j)}(t)\right\rangle \\
& =\langle f(t), \phi(t)\rangle \forall \phi \in D_{L_{p}}(R) \quad[\text { by }(4)] .
\end{aligned}
$$

Thus $F(z)$ is indeed the Cauchy representation of $f \in\left(D_{L_{p}}(R)\right)^{\prime}$.

We construct an example of a generalised function with non-compact support, and by using Theorem 2, prove the existence of its Cauchy representation.

EXAMPLE 1. Let $f(t)=\sum_{n=1}^{\infty} a_{n} \delta(t-n)$ where $a_{n}$ are appropriate constants (for example $\left.\sum_{n=1}^{\infty}\left|a_{n}\right|<\infty\right)$ and $\delta(t)$ be the Dirac delta function. Then, Supp $f=\{1,2,3,4, \ldots\}$ is a non-compact set and therefore, $f \notin(E(R))^{\prime}$. However, for every $\phi \in D_{L_{p}}(R)$ $(1<p<\infty)$ the map

$$
\phi \longrightarrow\langle f, \phi\rangle=\sum_{n=1}^{\infty} a_{n} \phi(n)
$$

is well defined since $\phi(x) \rightarrow 0$ as $|x| \rightarrow \infty[2]$. Therefore, the series (9) is convergent for all $\phi \in D_{L_{p}}(R)$ and $f \in\left(D_{L_{p}}(R)\right)^{\prime}$. According to Theorem 2, the Cauchy representation of the generalised function $f$ exists and is given by

$$
F(z)=\sum_{n=1}^{\infty} \frac{1}{2 \pi i} \frac{a_{n}}{(n-z)}
$$




\section{Probability distribution}

Suppose that a random variable $x$ taking real values between $-\infty$ and $\infty$ may be characterised by a probability distribution $\Phi(t)$, where $\Phi(t)$ is defined as the probability that $x$ lies in the interval $(-\infty, t)$. Then, we have

$$
\begin{gathered}
\Phi(t) \leqslant 1 \text { for }-\infty<t<\infty \\
\Phi\left(t_{1}\right) \leqslant \Phi\left(t_{2}\right) \text { for } t_{1}<t_{2}
\end{gathered}
$$

and $\lim _{t \rightarrow \infty} \Phi(t)=1$.

The quantity

$$
f(t)=\frac{d}{d t} \Phi(t)
$$

is called the probability density. In certain cases (that is, the case of discontinuous probability distribution) the derivative in (11) does not exist in the ordinary sense. However, the probability density (11) defines a generalised function on some space of test functions. For example, suppose that it is certain that the random variable $x$ takes the value $x_{0}$. Then,

Thus

$$
\Phi(t)= \begin{cases}0 & \text { for } t<x_{0} \\ 1 & \text { for } t>x_{0}\end{cases}
$$

$$
\Phi(t)=H\left(t-x_{0}\right)
$$

where $H$ is the Heaviside step function. In this case the probability density $f(t)$ does not exist in the ordinary sense. If, however, we admit generalised functions, then,

$$
f(t)=\frac{d}{d t} H\left(t-x_{0}\right)=\delta\left(t-x_{0}\right)
$$

is the Dirac delta function.

As a matter of fact every probability density $f(t)$ belongs to a certain space of generalised functions. We prove this fact in the following theorem.

THEOREM 3. Every probability density $f(t)$ defines a generalised function on the space $D_{L_{p}}(R)(1<p<\infty)$ of test functions.

Proof: For any $\psi \in D_{L_{p}}(R) \quad(1<p<\infty)$

$$
\langle f(t), \psi(t)\rangle=\left\langle\frac{d \Phi}{d t}, \psi(t)\right\rangle=(-1) \int_{-\infty}^{\infty} \Phi(t) \frac{d \psi}{d t} d t
$$


Since the map $\psi \rightarrow d \psi / d t$ is continuous injection $[5]$ on $D_{L_{p}}(R)(1<p<\infty)$ and $\Phi(t)$ is bounded, the integral (12) exists, that is, the linear operation $f(t) \rightarrow\langle f(t), \psi(t)\rangle$ is well defined. Moreover,

$$
\begin{aligned}
|\langle f(t), \psi(t)\rangle| & \left.\leqslant \int_{-\infty}^{\infty}\left|\frac{d \psi}{d t}\right| d t \quad \text { (because } \Phi(t) \leqslant 1\right) \\
& \leqslant C_{p}\|\psi\|_{L_{p}} \quad[2] .
\end{aligned}
$$

Therefore, $f(t)$ is bounded.

Consequently, $f(t)$ defines a generalised function on $D_{L_{p}}(R)(1<p<\infty)$. The following corollary is an immediate consequence of Theorems 2 and 3.

COROLlary 1. For every probability density $\phi(t)$, the Cauchy representation, as defined in (2), exists.

EXAMPLE 2. Suppose that a random variable takes values $t_{1}, t_{2}, t_{3}, \ldots$ with probabilities $p_{1}, p_{2}, p_{3}, \ldots, \sum_{j} p_{j}=1$. Then,

$$
\Phi(t)=\sum_{j} p_{j} H\left(t-t_{j}\right)
$$

The probability density $f(t)$ is the generalised derivative of $\Phi(t)$, that is,

$$
f(t)=\sum_{j} p_{j} \delta\left(t-t_{j}\right)
$$

In view of Theorem 2 and Theorem 3 proved earlier, $f(t)$ defines a generalised function on $D_{L_{p}}(R)(1<p<\infty)$ and the Cauchy representation of $f(t)$ equals

$$
\widehat{f}(z)=\frac{1}{2 \pi i} \sum_{j} p_{j} \frac{1}{t_{j}-z} .
$$

For example, the binomial probability distribution is given by:

$$
\Phi(t)=\sum_{j=1}^{n}\left(\begin{array}{l}
n \\
j
\end{array}\right) u^{j}(1-u)^{n-j} H(t-j) ;
$$

$u$ is a parameter and the random variable takes the values $1,2,3, \ldots, n$. The probability density is:

$$
f(t)=\sum_{j=1}^{n}\left(\begin{array}{l}
n \\
j
\end{array}\right) u^{j}(1-u)^{n-j} \delta(t-j)
$$


and the Cauchy representation of $f(t)$ is:

$$
\widehat{f}(z)=\sum_{j=1}^{n}\left(\begin{array}{c}
n \\
j
\end{array}\right) u^{j}(1-u)^{n-j} \frac{1}{j-z} .
$$

\section{REFERENCES}

[1] H.J. Bremermann, Distributions, Complex Variables and Fourier Transforms (Addison Wesley, 1965).

[2] A. Friedman, Generalized Functions and Partial Differential Equations (Prentice Hall, Englewood Cliffs, N.J., 1963).

[3] D. Mitrovic, 'A distributional representation of analytic functions', Math. Balkanica 2 (1972), 161-164.

[4] J.N. Pandey and M.A. Chaudhry, 'Hilbert transform of generalized functions and applications', Canad. J. Math. XXXV, No.3 (1983), 478-495.

[5] L. Schwartz, Theories des Distributions, Vol.I, II (Herman, Paris, 1957, 1959).

[6] A.H. Zemanian, Generalized Integral Transformations (Interscience Publishers, New York, 1968).

Department of Mathematical Sciences

KFUPM, Dhahran

Saudi Arabia 